\title{
Larvicultura do Mandi-amarelo Pimelodus maculatus Lacépède, 1803 (Siluriformes: Pimelodidae) em Diferentes Densidades de Estocagem nos Primeiros Dias de Vida ${ }^{1}$
}

\author{
Ronald Kennedy Luz², Evoy Zaniboni Filho ${ }^{3}$
}

\begin{abstract}
RESUMO - Este trabalho foi realizado na Estação de Piscicultura de São Carlos (EPISCar), São Carlos, SC. O efeito da densidade de estocagem de Pimelodus maculatus na larvicultura foi avaliado em pós-larvas alimentadas diariamente com zooplâncton selvagem na proporção de 300 organismos/pós-larva/dia e submetidas aos seguintes tratamentos: T1 - 5 pós-larvas/litro; T2 - 15 pós-larvas/litro; T3 - 30 pós-larvas/litro. No terceiro e quinto dias de experimento, foi avaliada a taxa de sobrevivência. Decorridos três dias de experimento, não foi observada influência da densidade na sobrevivência, embora, no quinto dia de experimento, houvesse maior sobrevivência na menor densidade testada, com valores médios de sobrevivência final de T1 (35,6\%), T2 (28,9\%) e T3 (20,6\%). Os maiores valores de peso foram encontrados no tratamento de menor densidade, sendo os valores de comprimento semelhantes entre os tratamentos. O tratamento contendo 30 pós-larvas/L apresentou as menores taxas de sobrevivência e a maior produtividade final.
\end{abstract}

Palavras chave: densidade de estocagem, larvicultura, mandi-amarelo, Pimelodus maculatus, pós-larva

\section{Larviculture of Yellow Mandi Pimelodus maculatus Lacépède, 1803 (Siluriformes: Pimelodidae) at Different Stocking Densities during the First Days of Life}

\begin{abstract}
The effect of stocking density on the larviculture of Pimelodus maculatus post-larvae was evaluated. The experiment was conducted at the Sao Carlos hatchery (EPISCar), SC. Post-larvae were daily fed zooplankton at the rate of 300 organisms/post-larvae/ day at the following densities: T1. 5 post-larvae/L; T2. 15 post-larvae/L; and T3. 30 post-larvae/L. Survival rates were evaluated on the third and fifth day of the experiment. After three days, density did not affect survival rates; however, on the fifth day survival rate was higher for the lowest density. Average survival rates at the end of the experiment were 35.6, 28.9 and 20.6\% for T1, T2 and T3, respectively. Post-larvae average weight was also higher at the lowest density, but average length did not differ significantly among treatments. At density of 30 post-larvae/L, post-larvae survival rate was the lowest, but final productivity was the highest.
\end{abstract}

Key Words: stocking density, larviculture, yellow mandi, Pimelodus maculatus, post-larvae

\section{Introdução}

O mandi-amarelo (Pimelodus maculatus), espécie de ampla distribuição geográfica e de grandes variações cromáticas e até estruturais (Santos, 1954), pode ser encontrado na Amazônia, Guianas, Venezuela, Peru, Bolívia, Paraguai, Argentina, Bacia do Paraná, do Prata, Rio Uruguai e Rio Iguaçu (Godoy, 1987). É uma espécie abundante e importante na pesca comercial (Lolis et al., 1996), com ótima aceitação pelo mercado consumidor (Souza \& Torres, 1984).

De hábito alimentar onívoro com tendência à ictiofagia (Basile-Martins, 1978; Souza, 1982; Menin \& Mimura, 1991), o mandi-amarelo apresenta ampla plasticidade da dieta.

Um dos maiores problemas enfrentados na produção de alevinos de espécies nativas brasileiras está relacionado à fase de larvicultura, que consiste na busca das condições ideais de cultivo para proporcionar maiores taxas de sobrevivência. Os procedimentos comumente empregados para outras espécies ainda apresentam o problema de predação de larvas quando soltas diretamente em tanques de cultivo, reduzindo significativamente a produção final de alevinos (Basilie-Martins, 1984).

A densidade de estocagem é um importante fator a ser considerado na larvicultura de peixes, uma vez que pode afetar o crescimento dos peixes (Hecht \& Uys, 1997) e influir na alimentação (Bonga, 1997) e no comportamento das larvas (Mackinnon, 1985); além disso, baixas densidades podem levar ao inadequado aproveitamento do espaço (Jobling, 1994).

A densidade de estocagem a ser utilizada depende da espécie a ser criada, das condições de cultivo,

\footnotetext{
1 Projeto financiado pela GERASUL/MAESA.

${ }^{2}$ Engenheiro Agrônomo, Mestrando em Aqüicultura/UFSC, Bolsista/CAPES. E.mail: luzrk@yahoo.com

${ }^{3}$ Bolsista CNPq - Professor Adjunto - Depto. Aqüicultura/UFSC.Rod. SC 406, n. 3532, Lagoa do Peri, Cep: 88049-900, Cx.: 476.

E.mail: zaniboni@cca.ufsc.br
} 
do tipo de alimentação, do manejo adotado e do tamanho dos peixes, entre outros fatores. Por esses motivos, tem sido difícil estabelecer a densidade ideal para o cultivo das diferentes espécies de peixes brasileiros em condições de laboratório. De acordo com Zaniboni Filho (2000), o cultivo intensivo de póslarvas, em condições de laboratório, pode ser realizado pelo período de 7 a 10 dias, sendo recomendadas densidades entre 15 e 30 pós-larvas/L, com a inclusão de alimento natural ou artificial e controle total da qualidade da água.

Dessa forma, este experimento visou avaliar diferentes densidades de estocagem no cultivo de pós-larvas de Pimelodus maculatus nos primeiros dias de vida.

\section{Material e Métodos}

Este trabalho realizou-se na Estação de Piscicultura de São Carlos (EPISCar), localizada no município de São Carlos, SC.

As pós-larvas foram obtidas por intermédio de reprodução induzida de matrizes selvagens, mantidas durante o período de um ano em cativeiro e alimentadas com ração comercial extrusada.

Após o processo de fertilização a seco, foi acompanhado o desenvolvimento larval, para determinar o momento em que as larvas apresentassem movimentos natatórios horizontais. Neste momento, uma amostra de 15 pós-larvas foi fixada em formol $4 \%$ tamponado para posterior biometria. As pós-larvas foram sifonadas para bacias plásticas, contadas individualmente e transferidas para cubas de 3 litros, com sistema de aeração contínua e revestidas externamente com lona plástica preta.

As pós-larvas foram submetidas aos seguintes tratamentos:

T 1.5 pós-larvas/L;

T 2. 15 pós-larvas/L;

T 3.30 pós-larvas/L.

Cada tratamento foi composto de três repetições.

A alimentação consistiu de zooplâncton silvestre coletado diariamente com o auxílio de rede plâncton com malha de $63 \mu \mathrm{m}$, de tanque externo de terra previamente adubado com cama de frango, na proporção de $700 \mathrm{~g} / \mathrm{m}^{2}$. O plâncton foi peneirado em tela de $200 \mu \mathrm{m}$ e concentrado em béquer de $500 \mathrm{~mL}$. Em seguida, foi realizada a quantificação do número de organismos/mL através da realização da contagem de três amostras de $1 \mathrm{~mL}$ em placa escavada sob estereomicroscópio. O concentrado foi fornecido em cada unidade experimental na quantidade de 300 organismos/pós-larva/dia. A quantidade de alimento foi corrigida diariamente em função da mortalidade das pós-larvas. A alimentação foi oferecida quatro vezes ao dia ( $8,11,14$ e $18 \mathrm{~h})$.

Uma amostra de $5 \mathrm{~mL}$ do concentrado de zooplâncton foi coletada diariamente e fixada em formol $4 \%$ tamponado, para posterior análise qualitativa. A identificação dos organismos do zooplâncton foi feita com base em literatura especializada (Koste, 1978; Tundisi, 1983; Reid, 1985).

Uma vez por dia, foi realizada a limpeza das cubas por sifonamento, retirando-se a aeração para a precipitação do material em suspensão, sendo o volume retirado imediatamente reposto. A renovação diária foi de $50 \%$ do volume total das cubas e as pós-larvas mortas quantificadas.

No terceiro dia de experimento, procedeu-se à troca do volume total de água e realizou-se uma contagem individual das pós-larvas para se determinar a sobrevivência.

O mesmo procedimento foi adotado no quinto dia (último dia de experimento). Após este procedimento, as pós-larvas foram fixadas em formol $4 \%$ tamponado para posterior biometria.

Diariamente, às 8 e $18 \mathrm{~h}$, foram medidas a temperatura da água e a concentração de oxigênio dissolvido. $\mathrm{O} \mathrm{pH}$, alcalinidade e dureza foram medidos no primeiro dia de experimento, enquanto a concentração de amônia e nitrito foi avaliada no terceiro e quinto dias do experimento.

As pós-larvas foram medidas com o auxílio de um paquímetro. Para a determinação do peso, usou-se balança analítica Mettler AE 260 (Delta Range) com precisão de 0,1 mg. Papel secante retirou o excesso de líquido das pós-larvas. Os dados de peso e comprimento foram submetidos a uma análise de variância e as médias comparadas pelo teste Duncan, em nível de $5 \%$ de probabilidade.

Os dados de sobrevivência observados no terceiro e quinto dias foram submetidos à análise de regressão linear, com nível de confiabilidade de 95\%.

\section{Resultados e Discussão}

A qualidade da água, nos três tratamentos, foi semelhante, apresentando valores médios de temperatura entre $22,4 \pm 1,4$ e $29,6 \pm 1,7^{\circ} \mathrm{C}$, considerando o período da manhã e da tarde, respectivamente. Esta 
variação aconteceu de forma gradativa. A concentração de oxigênio dissolvido manteve-se acima de $6,5 \mathrm{mg} / \mathrm{L}$ durante o experimento.

$\mathrm{O}$ pH da água apresentou valor médio de 6,5 , enquanto a alcalinidade e a dureza foi de $40 \mathrm{mg} / \mathrm{L}$. Os níveis de amônia e nitrito foram inferiores a $0,5 \mathrm{e}$ $0,01 \mathrm{mg} / \mathrm{L}$, respectivamente. Esses valores sugerem que o sistema de cultivo adotado foi suficiente para a manutenção da qualidade da água.

A utilização de zooplâncton, em condições de laboratório, tem-se mostrado eficiente para a larvicultura de várias espécies como: a carpa comum (Cyprinus carpio) (Dabrowski, 1984), larvas de pacu (Piaractus mesopotamicus) e tambaqui (Colossoma macropomum) (Sipaúba-Tavares \& Rocha, 1994), larvas e alevinos do salmão do Atlântico (Salmo salar) (Holm, 1987), para três espécies de bagre africano (Adeyemo et al., 1994) e para o matrinxã (Brycon cephalus) (Lopes et al., 1994).

A utilização de zooplâncton silvestre pode introduzir patógenos e predadores (Adeyemo et al., 1994), fato que não foi observado no presente experimento. A análise qualitativa do zooplâncton fornecido diariamente é mostrada na Tabela 1.

Os rotíferos predominaram na composição do zooplâncton, com aproximadamente 75\% (44\% Keratella cochlearis, $29 \%$ de Brachionus angularis) e aproximadamente $25 \%$ de náuplios de ciclopóides.

Logo que as pós-larvas apresentaram movimentos natatórios horizontais, observou-se canibalismo já no primeiro dia do experimento e em todas as densidades.

Os valores de sobrevivência observados no terceiro dia foram semelhantes nas diferentes densidades, sendo sempre maiores que $50 \%$. No quinto dia, foi observada redução significativa $(\mathrm{P}<0,05)$ na so- brevivência dos peixes estocados na maior densidade (Figura 1). A densidade de 5 pós-larvas/L apresentou maiores valores de sobrevivência no terceiro dia $(80,0 \%)$ e ao final do trabalho $(35,6 \%)$. Lopes et al. (1996), testando duas densidades para a larvicultura do pintado (Peseudoplatystoma corruscans), alimentados com vários organismos vivos por cinco dias, observaram também a maior taxa de sobrevivência na menor densidade utilizada.

Zaniboni Filho \& Barbosa (1992) recomendam que as pós-larvas de jaú (Paulicea lutkeni) sejam mantidas em tanques aerados durante três dias, em baixas densidades de estocagem, sendo alimentadas com larvas forrageiras para garantir baixa mortalidade e ausência de canibalismo. Bastos Filho et al. (1996), utilizando densidades de 5 pós-larvas/L na larvicultura de pintado (Pseudoplatystoma corruscans), obtiveram sobrevivência de $32 \%$ no segundo dia de alimentação com rotífero de origem marinha.

A utilização de baixas densidades para a larvicultura de espécies que apresentam canibalismo foi avaliada por Behr \& Hayashi (1997), alimentando pós-larvas de pintado (Pseudoplatystoma corruscans) exclusivamente com Artemia, a uma densidade de 10 pós-larvas/L, e obtendo sobrevivência de $65,6 \%$ no nono dia de experimento.

Para a densidade de 15 pós-larvas/L, no presente experimento, foi observada a sobrevivência média de $28,9 \%$ no quinto dia de trabalho. Luz (2000), avaliando a utilização de diferentes dietas para a primeira alimentação de Pimelodus maculatus, com a mesma densidade, observou que a maior taxa de sobrevivência ao final de cinco dias de cultivo foi observada quando as pós-larvas foram alimentadas com náuplios de Artemia (39,3\%), sendo este alimento mais efici-

Tabela 1 - Análise qualitativa do zooplâncton fornecido diariamente na dieta de $P$. maculatus Table 1 - Qualitative analysis of zooplankton daily supplied on diets of P. maculatus

\begin{tabular}{|c|c|c|c|c|c|c|}
\hline $\begin{array}{l}\text { Grupo } \\
\text { Group }\end{array}$ & $\begin{array}{l}\text { Espécie } \\
\text { Species }\end{array}$ & $\begin{array}{l}1^{\mathrm{o}} \mathrm{dia} \\
1^{s t} d a y\end{array}$ & $\begin{array}{l}2^{\mathrm{o}} \mathrm{dia} \\
2^{\text {nd }} \text { day }\end{array}$ & $\begin{array}{l}3^{o} \text { dia } \\
3^{r d} d a y\end{array}$ & $\begin{array}{l}4^{\circ} \text { dia } \\
4^{\text {th }} d a y\end{array}$ & $\begin{array}{l}5^{\circ} \text { dia } \\
5^{\text {th }} \text { day }\end{array}$ \\
\hline Rotífera & Brachionus angularis & + & + & + & + & + \\
\hline \multirow[t]{3}{*}{ Rotifers } & Keratella cochlearis & + & + & + & + & + \\
\hline & Polyartra vulgaris & - & - & - & + & + \\
\hline & $\begin{array}{c}\text { Trichocerca } \mathrm{sp} \\
\text { náuplios de Ciclopóides }\end{array}$ & - & - & + & + & + \\
\hline Crustacean & nauplii of Cyclopoids & + & + & + & + & + \\
\hline
\end{tabular}

+organismo presente na dieta.

- organismo ausente na dieta.

+organism at diets.

- lack of organism at diets. 


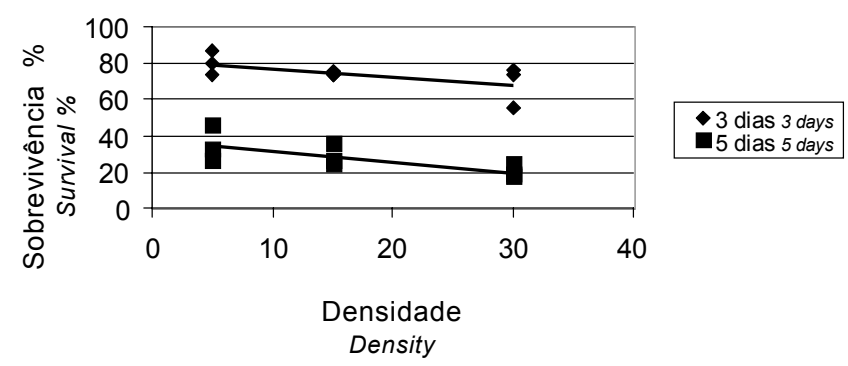

Figura 1 - Taxas de sobrevivência de pós-larvas de $P$. maculatus nos diferentes tratamentos, no terceiro e quinto dias de vida.

Figure 1 - Survival rate the post-larvae of $P$. maculatus in the different treatments in the third and fifth days of life.

ente que a utilização de ração e zooplâncton silvestre composto de $47 \%$ de copépodos, $47 \%$ de rotíferos e $6 \%$ de cladóceros.

A utilização de densidades elevadas tem sido utilizada com sucesso para espécies que não apresentam canibalismo, tais como o curimbatá (Prochilodus scrofa), sendo aconselhada a densidade de 40 pós-larvas/L (Cestarolli et al., 1997) e 50 póslarvas/L para três espécies de bagre africano (Adeyemo et al., 1994), com a obtenção de altas taxas de sobrevivência. Na tentativa de redução do canibalismo de Pimelodus maculatus cultivado em maior densidade (30 pós-larvas/L), Luz et al. (2001) realizaram a larvicultura em água limpa (cubas revestidas com lona preta) e água turva (água + argila), alimentando as pós-larvas com zooplâncton silvestre, e observaram valores semelhantes de sobrevivência e comprimento das pós-larvas depois de 5 dias, com valores médios de $12,63 \pm 3,1 \%$ e $8,3 \pm 0,6 \%$ de sobrevivência e 4,9 $\pm 0,2 \mathrm{~mm}$ e $5,1 \pm 0,3 \mathrm{~mm}$, para os dois tratamentos, respectivamente.

Foi observada diariamente, em todos os tratamentos, a presença de restos de pós-larvas parcialmente consumidas e pós-larvas inteiras mortas, que, sob análise microscópica, apresentavam mordidas na altura do vitelo, mostrando a ocorrência de canibalismo. O canibalismo ocorreu com maior freqüência no tratamento com maior densidade. A análise do trato digestório dos indivíduos mortos revelou grande quantidade de presas, mostrando que o zooplâncton oferecido foi ingerido pelas pós-larvas. Apesar disso, o manejo de alimentação e a composição do zooplâncton não foram eficientes no controle da ocorrência de canibalismo. Segundo Pienaar (1990), o canibalismo pode ser controlado por uma simples alteração na disponibilidade de alimento.

A menor taxa média de sobrevivência, no tratamento com 30 pós-larvas/L, produziu três vezes mais indivíduos neste tratamento que naquele com menor densidade e maior taxa de sobrevivência, possibilitando a obtenção de uma maior produtividade final de pós-larvas para as condições estudadas. Fato semelhante é relatado por Gomes (1998) no cultivo de matrinxã (Brycon cephalus).

$\mathrm{O}$ peso final das pós-larvas mantidas sobre a densidade de 5 pós-larvas/L foi superior $(\mathrm{P}<0,05)$ ao observado nas densidades superiores (Tabela 2), embora os valores de comprimento tenham sido semelhantes nos diferentes tratamentos. Diversos autores têm relatado a influência da densidade de estocagem sobre o crescimento dos peixes. Altas densidades mostraram-se favoráveis ao crescimento de juvenis do bagre africano (Clarias gariepinus) mantidos em tanques de concreto (Hecht \& Uys,

Tabela 2 - Valores de peso e comprimento final e inicial das pós-larvas de $P$. maculatus em diferentes densidades de estocagem

Table 2 - Weight values, final and initial length of $P$. maculatus for different stocking densities

\begin{tabular}{|c|c|c|c|}
\hline & $\begin{array}{l}5 \text { pós-larvas } / \mathrm{L} \\
5 \text { post-larvae } / L\end{array}$ & $\begin{array}{l}15 \text { pós-larvas/L } \\
15 \text { post-larvae/L }\end{array}$ & $\begin{array}{l}30 \text { pós-larvas/L } \\
30 \text { post-larvae } / L\end{array}$ \\
\hline Peso inicial (mg) & $0,15 \pm 0,05$ & $0,15 \pm 0,05$ & $0,15 \pm 0,05$ \\
\hline Initial weight ( $\mathrm{mg}$ ) & & & \\
\hline $\begin{array}{l}\text { Comprimento inicial }(\mathrm{mm}) \\
\text { Initial lenght }(\mathrm{mm})\end{array}$ & $4,3 \pm 0,2$ & $4,3 \pm 0,2$ & $4,3 \pm 0,2$ \\
\hline $\begin{array}{l}\text { Peso final }(\mathrm{mg}) \\
\text { Final weight }(\mathrm{mg})\end{array}$ & $0,25 \pm 0,1 \mathrm{a}$ & $0,21 \pm 0,1 b$ & $0,19 \pm 0,1 b$ \\
\hline $\begin{array}{l}\text { Comprimento final }(\mathrm{mm}) \\
\text { Final lenght }(\mathrm{mm})\end{array}$ & $4,9 \pm 0,2 \mathrm{a}$ & $4,9 \pm 0,3 \mathrm{a}$ & $5,0 \pm 0,1 \mathrm{a}$ \\
\hline
\end{tabular}

Letras diferentes, em linha, indicam diferença significativa no teste Duncan, a 5\% de significância.

Different letters in the line show significative difference by Duncan test $(P<.05)$. 
1997) e para o jundiá Rhamdia quelen (Piaia \& Baldisserotto, 2000), mas reduzem o crescimento de larvas de Clarias gariepinus em aquários (Kaiser et al., 1997) e alevinos do bagre do canal (Ictalurus punctatus) em tanques de terra (Esquivel et al., 1997). Zaniboni Filho \& Barbosa (1992), avaliando a larvicultura de várias espécies de peixes brasileiros em tanques de terra, perceberam que o crescimento em comprimento e peso dos alevinos era inversamente proporcional à densidade de estocagem.

O Pimelodus maculatus é uma espécie que apresenta canibalismo desde o momento em que as pós-larvas passam a nadar ativamente na horizontal. Este fato é um dos principais fatores de entrave para a produção de alevinos de espécies nativas brasileiras, estando a ocorrência de canibalismo relacionada à densidade de estocagem e à alimentação.

\section{Conclusões}

A utilização de baixa densidade de estocagem das pós-larvas de Pimelodus maculatus (5 pós-larvas/L) proporcionou maior taxa de sobrevivência e indivíduos com maiores valores de peso, apresentando menor produtividade final (número final de pós-larvas três vezes inferior) à densidade de 30 pós-larvas/L.

\section{Literatura Citada}

ADEYEMO, A.A.; OLADOSU, G.A.; AYINLA, A.O. Growth and survival of fry African catfish species, Clarias gariepinus Burchell, Heterobranchus bidorsalis Geoffery and Heteroclarias reared on Moina dubia in comparison with other first feed sources. Aquaculture, v.119, p.41-45, 1994.

BASILE-MARTINS, M.A. Comportamento e alimentação de Pimelodus maculatus Lacépède, 1803 (Osteichthyes, Siluriformes, Pimelodidae). São Paulo: Universidade de São Paulo, 1978. 143p. Tese (Doutorado em Recursos Naturais) Universidade de São Paulo, 1978.

BASILE-MARTINS, M.A. Criação de organismos para alimentação de larvas de peixes. In: SIMPÓSIO BRASILEIRO DE AQÜICULTURA, 1984, São Carlos. Anais... São Carlos, 1984. p.97-100.

BASTOS FILHO, R.A.; SENHORINI, J.A.; RIBEIRO, L.P. Estudos preliminares da larvicultura intensiva do pintado Pseudoplatystoma corruscans (Agassiz, 1829), (Pisces, Pimelodidae). In: SIMBRAq - SIMPÓSIO BRASILEIRO DE AQÜICULTURA, 1996, Sete Lagoas, MG. Resumos... Sete Lagoas: 1996. p.110.

BEHR, E.R.; HAYASHI, C. Alimentação de larvas de Pseudoplatystoma corruscans (Agassiz, 1829) em bandejas berçário durante o período crítico. In: XIIEBI - ENCONTRO BRASILEIRO DE ICTIOLOGIA 7., 1997, São Paulo. Resumos... São Paulo: 1997. p.51.

BONGA, S.E.W. The stress response in fish. Physiological Reviews, v.77, n.3, p.591-625, 1997.
CESTAROLLI, M.A.; PORTELLA, M.C.; ROJAS, N.E.T. Efeito do nível de alimentação e do tipo de alimento na sobrevivência e no desempenho inicial de larvas de Curimbatá Prochilodus scrofa (STEINDACHNER, 1881). Boletim do Instituto de Pesca, v.24, n. único, p.119-129, 1997.

DABROWSKI, K.; BARDEGA, R. Mouth size and predicted food size preferences of larvae of three cyprinid fish species. Aquaculture, v.40, p.41-46, 1984.

ESQUIVEL, B.M.; ESQUIVEL, J.R.; ZANIBONI FILHO, E. Effects of stocking density on growth of channel catfish, Ictalurus punctatus, fingerlings in Southern Brazil. Journal of Applied Aquaculture, v.7, n.3, p.1-6, 1997.

GODOY, M.P.D.E. Peixes do Estado de Santa Catarina. Florianópolis: Universidade Federal de Santa Catarina, 1987. $572 \mathrm{p}$.

GOMES, L.C. Sistema semi-intensivo para criação de larvas de Brycon cephalus. Panorama da aqüicultura, p.15-20, 1998.

HECTH, T.; UYS, W. Effect of density on the feeding and agressive behaviour in juvenile African catfish (Clarias gariepinus). South African Journal of Science, v.93, p.537-541, 1997.

HOLM, J.C. Atlantic salmon start-feeding with live zooplankton: Pressure shock treatment to increase prey availability. Aquaculture Engineering, v.6, p.1-14, 1987.

JOBLING, M. Fish bioenergetics. London: Chapman \& Hall, 1994. 294p.

KAISER, H.; WEYL, O.; HECTH, T. Observations on agnostic behaviour of Clarias gariepinus larvae and juveniles under different densites and feeding frequencies in a controlled environment. Journal of Applied Ichthyology, v.11, p.2536,1995 .

KOSTE, W. Rotatoria. die radertiere mitteleuropas, Uberordnung Monogononta. Berlin Gebruder Borntrager, 1978. 673p.

LOLIS, A.A.; ANDRIA, I.F. Alimentação de Pimelodus maculatus Lacépède 1803 (Siluriformes, Pimelodidae), na planície de inundação do Alto Rio Paraná, Brasil. Boletim do Instituto de Pesca, v.23, n.único, p.187-202, 1996.

LOPES, R.N.M.; FREIRE, R.A.B.; VICENSOTTO, J.R.M. et al. Alimentação de larvas de surubim Peseudoplatystoma corruscans (Agassiz, 1829) em laboratório na primeira semana de vida. Boletim Técnico CEPTA, v.9, p.11-29, 1996.

LOPES, R.N.M.; SENHORINI, J.A.; SOARES, M.C.F. Crescimento e sobrevivência de larvas de matrinxã Brycon cephalus Gunther, 1869, (Pisces, Characidae) sob diferentes dietas alimentares. Boletim Técnico CEPTA, v.7, p.41-48, 1994.

LUZ, R.K. Larvicultura do mandi-amarelo Pimelodus maculatus (Lacépède 1803): desenvolvimento embrionário, larval e primeira alimentação. Florianópolis: Universidade Federal de Santa Catarina, 2000. 47p. Dissertação (Mestrado em Aqüicultura) - Universidade Federal de Santa Catarina, 2000.

LUZ, R.K.; REYNALTE, D.A.T.; ZANIBONI FILHO, E. Influência da turbidez nos primeiros dias de vida do mandi-amarelo Pimelodus maculatus. In: ENCONTRO BRASILEIRO DE ICTIOLOGIA, 14., 2001., São Leopoldo. Resumos... São Leopoldo: 2001. (CD-ROM)

MACKINNON, M.R. Barramundi breeding and culture in Thailand. Sohgkhla: Queensland Department of Primary Industries Study Tour Report, 1982.

MENIN, E.; MIMURA, O.M. Anatomia da cavidade bucofaringeana de Pimelodus sp. (Siluriformes, Pimelodidae). Revista Ceres, v.38, p.218, p.286-304, 1991.

PIAIA, R.; BALDISSEROTTO, B. Densidade de estocagem e crescimento de alevinos de jundiá Rhamdia quelen (QUOY \& 
GAIMARD, 1824) fingerlings. Ciência Rural, v.30, n.3, p.509-513, 2000.

PIENAAR, A.G. A study of coeval sibling cannibalism in larval and juvenile fishes and its control under culture conditions. Grahamstown: Rhodes University, 1990. 162p. (Masters thesis) - Rhodes University, 1990.

REID, J.W. Chave de identificação para espécies continentais sulamericanas de vida livre da Ordem Cyclopoida (Crustacea, Copepoda). Boletim de Zoologia da USP, v.9, p.17-143, 1985.

SANTOS, E. Peixes de água doce. Rio de Janeiro: Briguit, 1954. $270 p$.

SIPAÚBA-TAVARES, L.H.; ROCHA, O. Sobrevivência de larvas de Piaractus mesopotamicus (Holmberg, 1887) (Pacu) e Colossoma macropomum (Cuvier, 1818) (Tambaqui), cultivadas em laboratório. Biotemas, v.7, n.1/2, p.46-56, 1994.

SOUZA, M.R.F. Observações sobre o espectro alimentar de Pimelodus maculatus Lacépède, 1803 (Osteichthyes, Siluriformes, Pimelodidae) da represa de Três Marias, MG. In: ENCONTRO ANUAL DE AQÜICULTURA DE MG, 1., 1982, Belo Horizonte. Resumos... Belo Horizonte: Associação Mineira de Aqüicultura, 1982. p.12.
SOUZA, M.R.F.; TORRES, G.E. Alimentação natural, especificidade alimentar e capacidade malacófaga do Pimelodus maculatus Lacépède, 1803 (Osteichthyes, Siluriformes, Pimelodidae) da represa de Três Marias, MG. In: ENCONTRO ANUAL DE AQÜICULTURA DE MG, 3., 1984, Igarapé, MG. Resumos... Igarapé: Associação Mineira de Aqüicultura, 1984. p.16-17.

TUNDISI, T.M. Tipologia de reservatórios do Estado de São Paulo - Ecologia do zooplâncton e do fitoplâncton. São Carlos: Universidade Federal de São Carlos, 1983. 35p. (Relatório Técnico Científico)

ZANIBONI FILHO, E. Larvicultura de peixes de água doce. Informe Agropecuário, v.21, n.203, p.69-77, 2000.

ZANIBONI FILHO, E.; BARBOSA, N.D.C. Larvicultura na CEMIG. In: ENCONTRO ANUAL DE AQÜICULTURA DE MG, 10., 1992, Belo Horizonte. Anais... Belo Horizonte: 1992. v.10, p.36-42.

Recebido em: 25/05/01

Aceito em: 09/10/01 